\title{
A Multi-Criteria Based Approach to Prototyping Urban Road Networks
}

\author{
Atulya Nagar and Hissam Tawfik \\ Intelligent and Distributed Systems Research Laboratory \\ Liverpool Hope University, Liverpool, UK
}

\author{
nagara@hope.ac.uk tawfikh@hope.ac.uk
}

\begin{abstract}
This paper presents an approach for analysing and prototyping urban road network routes based on multiple criteria, such as spatial quality, transportation cost and aesthetics, in order to evaluate the quality of people's use of road networks. A prototype is developed to analyse routes and accessibility in road layouts from a number of design and user related perspectives. A case study is used to demonstrate the concept of multi-criteria assessment of road networks.
\end{abstract}

Keywords: road networks, multi-criteria, simulation.

\section{Introduction}

Space layout and urban road network design presents a critical challenge as a result of increasing levels of urbanisation and road traffic. Urban planners have always aimed at optimizing the road network design to meet transportation cost, safety, land use, aesthetic and environmental considerations. With the rapid growth in traffic patterns and space utilisation, there is a growing need for a tool to design and evaluate urban road networks. This can be accomplished by efficient computer simulation techniques to aid the analysis of routes traffic networks from various design and user related objectives.

This research aims to investigate the application of computer modelling and graphical simulation techniques for supporting the evaluation of routes in road networks from a range of design and user related perspectives, and present a multi-criteria based approach to prototyping road networks. In an earlier part of this work (Khan et al., 2006), which focused on road network analysis of Liverpool City, it was obtained that a multi-criteria based analysis of urban road network routes and spatial layouts enables local accessibility of the road network for the set criteria, global accessibility of the road network, and the finding of optimum path between two points in a network.

Multi-criteria analysis has been defined in different ways. For the purpose of this work, we identify a common definition by (Roy, 1996), which views multi-criteria analysis as a decision-aid

Material published as part of this publication, either on-line or in print, is copyrighted by the Informing Science Institute. Permission to make digital or paper copy of part or all of these works for personal or classroom use is granted without fee provided that the copies are not made or distributed for profit or commercial advantage AND that copies 1) bear this notice in full and 2) give the full citation on the first page. It is permissible to abstract these works so long as credit is given. To copy in all other cases or to republish or to post on a server or to redistribute to lists requires specific permission and payment of a fee. Contact Publisher@InformingScience.org to request redistribution permission. and a mathematical tool that allows the comparison of different alternatives or scenarios according to many criteria, often contradictory, in order to guide toward a 'good' decision. The decision maker has to choose from several options or alternatives and will generally have to be contented with a compromising solution. 


\section{Related Work}

Road network analysis and prototyping has been performed using different technologies and algorithms, one of which is Space Syntax (Hillier, 1996), a set of analytical and computational tools for the analysis of urban systems. Space syntax can be considered a form of study of geometrical accessibility where a functional relationship is made, or attempted, between the structure of the city and the social, economical and environmental dimensions (Bera \& Claramunt, 2004). According to the paper, a number of space syntax empirical studies have been devoted to the exploration of the complexity and configuration of urban space by searching for a computational representation of a form of geometric order. Space syntax has been successfully applied to relationship between the structure of the city and criminality, way-finding and pollution (Peponis et al., 1990; Ortega et al., 2001). According to the space syntax concepts, the accessibility of the spatial layouts is based on the idea that some places or streets are more accessible than others, though the terms used for accessibility such as proximity, integration, connectivity, cost, effort or centrality differ (Porta et al., 2005).

Jiang and Claramunt (2003) proposed a topological analysis of large urban street networks based on a computational and functional graph representation. This representation gives a functional view in which vertices represent named streets and edges represent street intersections. A range of graph measures including street connectivity, average path length and clustering coefficient are computed for structural analysis. They applied validations to three cities and showed that large urban street networks form small-world networks but do not exhibit scale-free property.

The concept of network analysis uses Dijkstra algorithm (Dijkstra, 1959) to visualize the network pattern of street structure. In the work of Napong and Fujii (2004), the street patterns of two cities were analyzed on the basis of two different methods. Space syntax's Axial Map was applied to evaluate syntactic intelligibility and the degree of natural movement at both towns. Nophaket (2004) proposed graph geometry for detecting the minimum path structures. However, most researchers argue that that space syntax cannot be generalized across different cultures, archetypes and scales of street pattern.

Lee and Lee (1996) implemented inexact strategy and planning in route planning in Taipei City. Chakhar and Martel (2003) presented a strategy for integrating Geographical Information System (GIS) and multi-criteria analysis, and also proposed a design for a spatial decision support system. However, very little research has been carried to formulate prototypes of road networks and develop computer-aided tools to enable urban planners to model road network routes and urban planning from multiple perspectives.

\section{A Multi-Criteria Route Evaluation Prototype}

We designed a graphical simulation prototype to support multi-objective route evaluation of traffic networks. Our prototype uses a graphical user interface system to enable a user to load, initialise and modify an existing road traffic layout. It then determines the best route between two points on the network designated as the start and destination points, based on a combination of distance, safety, comfort and view criteria. Ultimately it allows for the evaluation of the level of accessibility of the local and global road network. The prototype consists of three layers: Visualisation layer, Road layout generation layer and Road layout evaluation layer, which are described below.

- $\quad$ Visualisation Layer: This layer is the interface between the user and the system where the user inputs the requirements which consist of loading the map, setting the weights for the criteria and the starting and destination points for a given path. 
- $\quad$ Road Layout Generation Layer: This layer converts the road map into the connected graph by using junctions as nodes and roads as links, and stores information about distance, safety, comfort and view for all the roads in the network.

- $\quad$ Road Layout Evaluation Layer: This layer is used to obtain the optimum paths in the road network. It uses Dijkstra algorithm (Dijkstra, 1959) to obtain optimum accessibility of the road networks. This layer uses the data obtained from the road layout generation layer and cost function to evaluate accessibility based on multi-objective criteria. The outcome of the multi-criteria analysis is represented visually in this layer, and is used to analyse the local and global accessibility of the road network. Local accessibility of a given road or path can be determined by calculating the mean value of the cost of all roads in the network from the given road, while global accessibility of the whole road network is a result of the average values of the local accessibility of all roads in the network.

The application is developed using the Java programming language and uses applets to construct the user interface (the visualization layer). A typical prototyping scenario starts by loading the road map of the location whose road network is to be evaluated into the prototype. The road map is converted into a connected graph using the nodes and links on the map. The junctions are represented as nodes and roads as links. A data file is used to store information for every road in the road map, such as distance between the connecting node pair, safety of the road, comfort, and view. This information is heuristically determined based on information about the number of turns, number of signals, number of accidents or thefts, etc. The attributes of the road, such as safety, comfort and view are provided between the scales ranging from 1 to 10 , where 1 represents the best case and 10, the worst. Figures $1 \mathrm{a}$ and $1 \mathrm{~b}$ show a graphical representation of a road map and the node-arc matrix format in which the data for the map is stored. In this matrix, the five columns represent the heuristically generated values for the start node, the destination node, the journey's distance, level of safety, level of comfort, and view (aesthetics) level, respectively.

The cost function is designed with the objective of determining the cost of the road according to the multi objective criteria. The cost function is applied to all the possible roads to reach the destination from a starting point. The selection of optimum path is based on the cost of path, denoted as $\mathrm{C}_{\mathrm{f}}$.

$$
\mathrm{C}_{\mathrm{f}}=\sum_{i=1}^{n} \mathrm{C}_{\mathrm{i}} * \mathrm{~W}_{\mathrm{i}}
$$

Where $\mathrm{n}$ is the number of criteria (safety, comfort, view), $\mathrm{C}_{\mathrm{i}}$ is the cost of the road for criterion $\mathrm{i}$, $\mathrm{W}_{\mathrm{i}}$ is the weight of criterion i provided by the user.

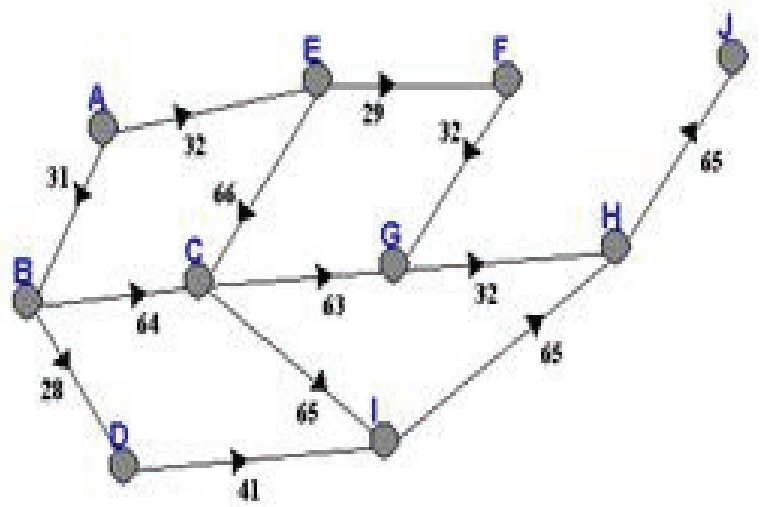

Figure 1a: Link-node representation of a road map

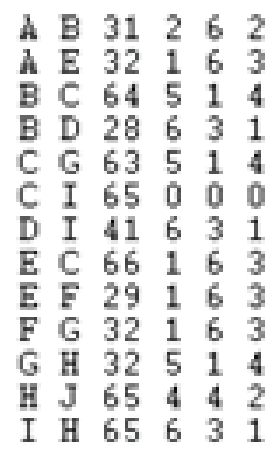

Figure 1b: Node-arc Matrix 
Dijkstra's algorithm (Dijkstra, 1959) is used to choose the optimum path, i.e. the path with minimum cost. Dijkstra's algorithm solves the single source shortest path problem on a weighted, directed graph in which all edge weights are non-negative.

The input to the algorithm consists of a weighted directed graph $\mathrm{G}$, the road network converted to connected graph, which takes the roads as edges and junctions as nodes. Let $\mathrm{s}$ be the source node in $\mathrm{G}$ from where the user wants to travel, and $\mathrm{t}$, the destination point. Let $\mathrm{V}$ denote the set of all nodes in graph $\mathrm{G}$. Each edge of the graph is an ordered pair of vertices $(\mathrm{u}, \mathrm{v})$ representing a connection from node $u$ to node $v$. The set of all edges is denoted $\mathrm{E}$. Weights of edges are given by a weight function $\mathrm{w}: \mathrm{E} \rightarrow[0, \infty]$; therefore $\mathrm{w}(\mathrm{u}, \mathrm{v})$ is the non-negative cost of moving from node $\mathrm{u}$ to node $\mathrm{v}$. The cost of an edge is determined by using the cost function. The cost of a path between two nodes is the sum of costs of the edges in that path. For a given pair of nodes $s$ and $t$ in $\mathrm{V}$, the algorithm finds the path from $\mathrm{s}$ to $\mathrm{t}$ with lowest cost. It is also used for finding costs of minimum cost paths from a single node $\mathrm{s}$ to all other nodes in the graph.

Dijkstra's algorithm with directed graph $\mathrm{G}=(\mathrm{V}, \mathrm{E})$, maintains a set $\mathrm{S}$ of vertices whose final shortest path weights from the source $s$ have already been determined. The algorithm repeatedly selects the node $\mathrm{u} \in \mathrm{V}-\mathrm{S}$ with the minimum cost path estimate, adds $\mathrm{u}$ to $\mathrm{S}$ and relaxes all edges leaving $\mathrm{u}$. Hence an optimum path is determined by calculating the cost of every road using cost function and finding the path with lowest cost using Dijkstras algorithm.

\section{Road Network Analysis Scenario}

Our simulation prototype has been designed to analyse the road network layouts in terms of their accessibility based on multi-criteria. This is illustrated using a case study based on Liverpool city centre's road network (Khan et al., 2006).

To analyse the road network design according to different criteria, the weights are assigned to each of the criteria, such as distance, safety, comfort and aesthetics. Based on these criteria the following analysis is obtained

- Local accessibility of the road network for the set criterion,

- Global accessibility of the road network and

- $\quad$ Optimum path between two points based on multi-criteria

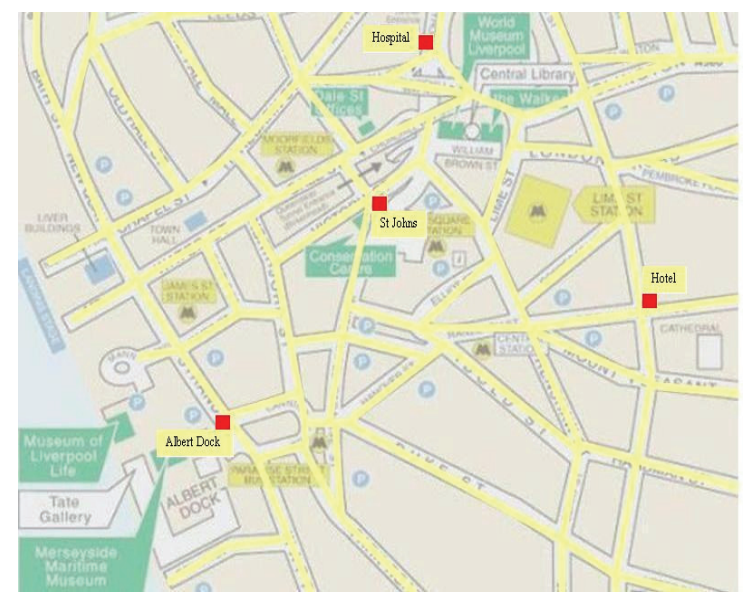

Figure 2a: Liverpool city centre map

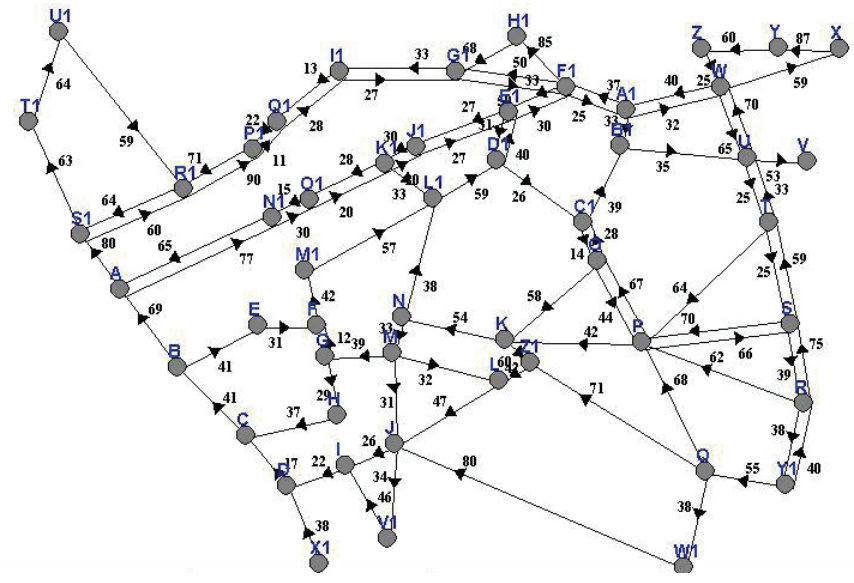

Figure 2b: Link-node Graph for Liverpool city centre 
Various scenarios are described to demonstrate the use of the prototype. The road network map of Liverpool city centre is converted into a connected graph using the nodes and links on the map. The junctions are represented as nodes and roads as links. Figure 2a shows the map of Liverpool city centre, and Figure $2 \mathrm{~b}$ shows the corresponding connectivity graph. The values for the criteria are predefined for different roads. See Figure 1.

The map highlights four locations - Hotel, the Albert Dock, St Johns and the Royal Hospital chosen to illustrate the multi-criteria based road network analysis. There is more than one path from a source location to chosen destination location. The optimum path obtained by the network analysis is based on the criteria and weights specified.

Using equal weights for the various criteria - distance, safety, view and comfort, a multi-criteria analysis for a path from Hotel to Albert Dock is obtained. Figures 3a to $3 \mathrm{c}$ show the optimum path, local accessibility and global accessibility using equal weights.

In a second scenario the same set of multi criteria - distance, safety, view and comfort are assigned different weights to account for preferences. To analyse the road network based on the high aesthetic appeal from Hotel to Alert Dock, the weight for view criteria is set to high. The optimum path after analysis is shown in figures $4 \mathrm{a}$ to $4 \mathrm{c}$. The local accessibility and global accessibility based on high aesthetic appeal are indicated in figures $4 \mathrm{~b}$ and $4 \mathrm{c}$.

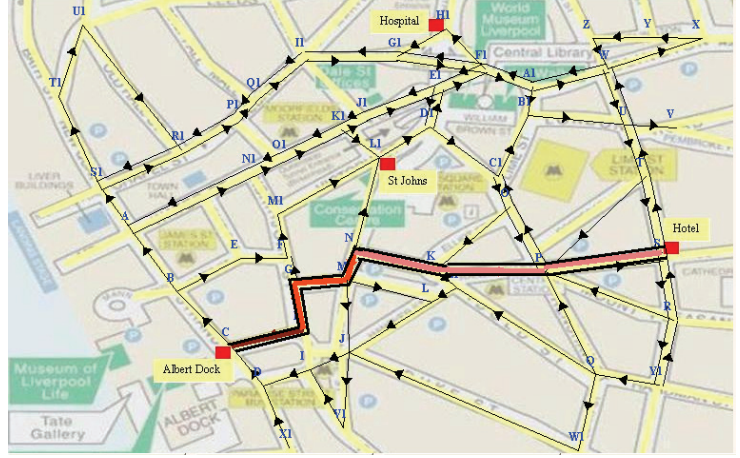

Figure 3a: Path from Hotel to Albert Dock for multi criteria analysis with equal weights

Low accessibility

High accessibility

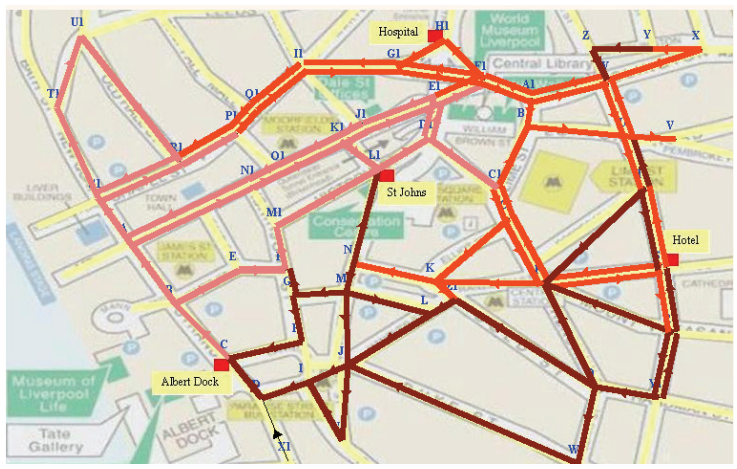

Figure 3b: Local accessibility for multi criteria analysis with equal weights



Figure 4a: Path from Hotel to Albert Dock with high aesthetic appeal

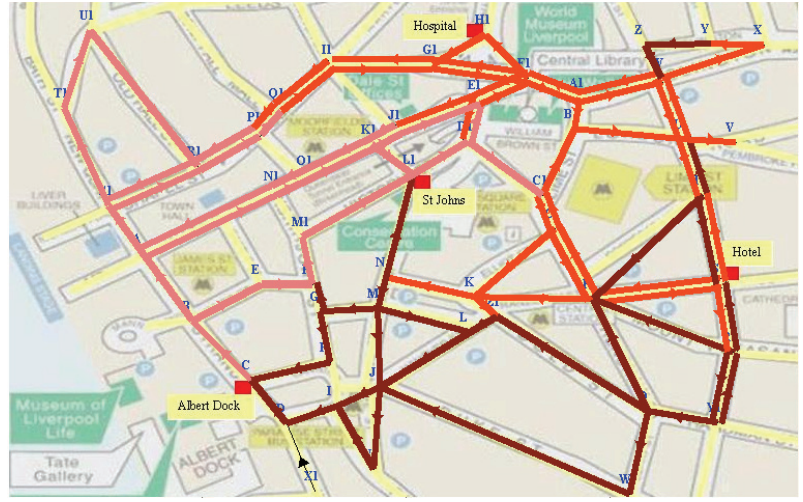

Figure 4b: Local accessibility of Albert Dock with high aesthetic appeal 


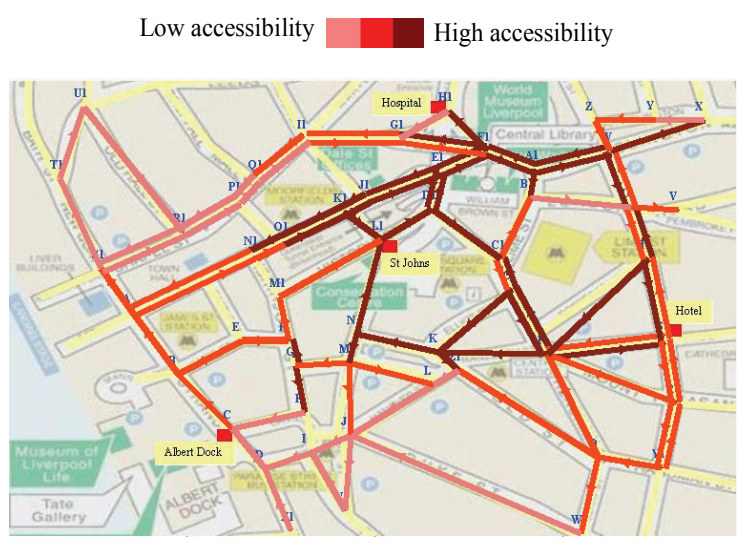

Figure 3c: Global accessibility for multi criteria analysis with equal weights

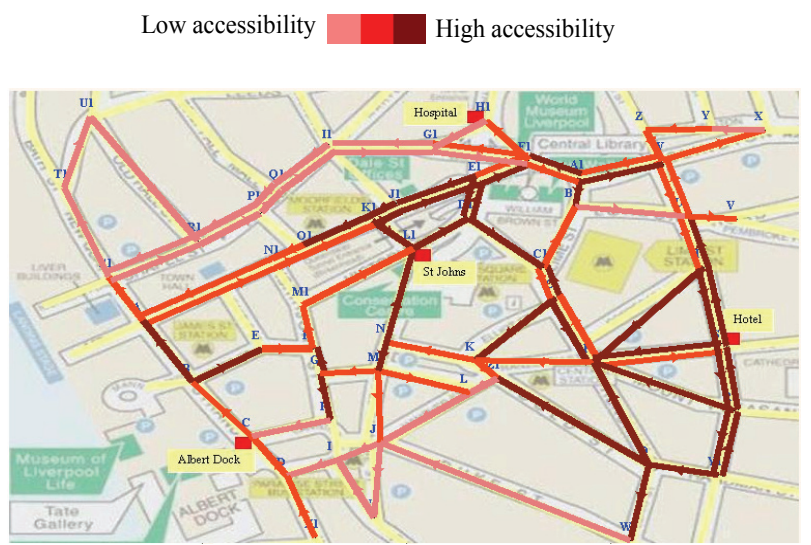

Figure 4c: Global accessibility of Albert Dock with high aesthetic appeal

The case study demonstrates the concept of accommodating for more than one criterion when considering route and road network analysis from design and user related perspectives. The criteria considered include the quality and aesthetics of the journey and levels of safety and comfort in addition to the conventional travel time, distance and cost. The results demonstrate that the path chosen from source to destination points, local accessibility and global accessibility will vary based on the weights assigned to the criteria.

The criteria can be emphasised depending on the type of user. New visitors to a city, for example, would typically like to take the safest route to their destination. Tourists, generally, are more interested in aesthetics, and would go through routes that present the most beautiful views of a city. Habitual road users are interested in saving time and cost as well as comfort, and would always take the shortest routes and those with less number of turns and lower traffic density. Emergency services providers, such as ambulance drivers, fire fighters and the police would on the other handy be likely to prioritise short routes over other criteria.

\section{Conclusion and Future Work}

This work investigates the application of computer simulation and modeling techniques to the analysis, evaluation and optimization of road networks from multi-perspectives. Our prototype uses multi-criteria evaluation to determine the optimum path, based on a range of multiple criteria, such as distance, safety, comfort and view. Our multi-criteria based analysis and prototyping could potentially support urban planners with early evaluation of road network layout in terms of its time, cost and safety implications on road users and pedestrians, and to assist with the provision of enhanced road network layouts. Our future work will focus on research to accommodate for complex road scenarios and traffic restrictions, and will take into account other factors such as environment and business criteria.

\section{Acknowledgement}

The authors would like to thank S. Dasari for the contribution to the programming phase of this work. 


\section{References}

Béra, R. \& Claramunt, C. (2004). Can relative adjacency contribute to space syntax in the search for a structural logic of the city? Proceedings of 3rd International Conference on Geo-graphical Information Science, Springer Berlin / Heidelberg, LNCS, Vol. 3234/2004, 38-50.

Chakhar, S. \& Martel, J. (2003). Enhancing geographical information systems capabilities with multicriteria evaluation functions. Journal of Geographic Information and Decision Analysis, 7, No. 2.

Dijkstra, E.W. (1959). A note on two problems in connection with graphs. Numeriche Mathematik, 1, 269271.

Hillier, B. (1996). Space is the machine. Cambridge University Press.

Jiang, B. \& Claramunt, C. (2003). Topological analysis of urban street networks, environment and planning B: Planning and Design, 31 No. 1, 151-162.

Khan, A., Dasari, S, Tawfik, H. \& Nagar, A. (2006). A case study based investigation for multi-criteria spatial layout analysis. In E. Pollard (Ed.), Proceedings of First European Modelling Symposium. University College London, September 2006, 113-119.

Lee, H. \& Lee, C, (1996). Inexact strategy and planning-the implementation of route planning in Taipei city. Proceedings of the 1996 Asian Soft Computing in Intelligent Systems and Information Processing, Fuzzy Systems Symposium, Kenting, Taiwan, 308-313.

Nophaket, N \& Fujii, A. (2004). Syntactic and network pattern structures of city - Comparison of grid and meandering street patterns in Kyojima and Honjo. Journal of Asian Architecture and Building Engineering (JAABE), 3(2), 349-356,

O’Neill, J. (1991). Evaluating a conceptual model of architectural legibility. Environment and Behavior, 23, 259-284.

Ortega, A, Jiménez, E., Jiménez, C., Mercado, S., \& Estrada, C. (2001). Sintaxis espacial: Una herramienta para la evaluación de escenarios. Paper presented at the $X V$ meeting of the Mexican Society of Behavioral Analysis, México.

Peponis, J., Zimring, C., \& Choi, Y. (1990). Finding the building in way finding. Environment and Behavior, 22, 555-590.

Porta, S., Crucitti, P., \& Latora, V. (2005). The network analysis of urban streets: A primal approach. Environment and Planning B: Planning and Design, 33(5), 705 - 725.

Roy, B. (1996). Multicriteria methodology for decision analysis. Kluwer, Dordrecht.

Soltani, A. R., Tawfik H., \& Fernando T. (2002). A multi-criteria based path-finding application for construction site layouts. Proceedings of 6th International Conference on Information Visualisation, July 2002. London, UK. 


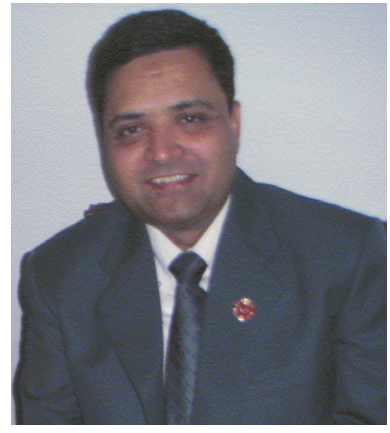

\section{Biographies}

Atulya Nagar leads the Intelligent and Distributed Systems (IDS) Research Group at the Liverpool Hope University. He earned his $\mathrm{PhD}$ in Mathematics from the University of York and has worked for several years at Brunel University, UK. He has multidisciplinary research interests in: systems engineering, bioinformatics, and applied mathematics.

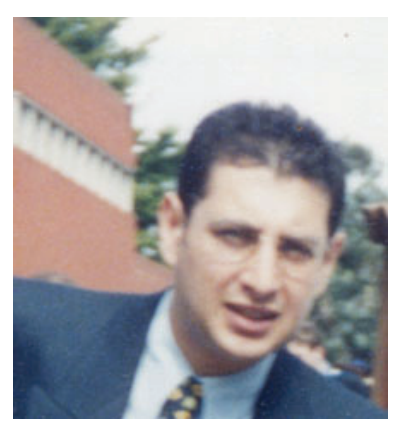

Hissam Tawfik is a Lecturer in Computing at Liverpool Hope University. He holds an MSc and PhD from in Computer Science and Engineering from University of Manchester (UMIST), UK. His research areas are in Artificial Intelligence and virtual reality applications. 